\title{
David Haslam
}

When the UK's National Institute for Clinical Excellence (NICE) was launched on April Fools' Day in 1999, some of its architects had doubts it would survive. Fourteen years on NICE is going strong, providing treatment guidance and drug cost-benefit analyses. Because many markets use UK drug pricing as a reference point, NICE's decisions also have a footprint far beyond the country. Now, the rebranded National Institute for Health and Care Excellence has its first new Chairman. David Haslam, a family doctor who was previously President of the British Medical Association and President of the Royal College of General Practitioners, took up oversight of the organization in April. He told Asher Mullard about his aims for NICE and the challenges of its new remit.

\section{Q What are your main goals for NICE in terms of drug cost-benefit appraisals? The life sciences industry is incredibly important and has brought a great deal to populations and individuals across the world. I see it as extremely important both from a commercial point of view and from a humanitarian point of view. But, like every health-care system in the world, the UK's National Health Service (NHS) faces almost infinite demand and has finite resources, which are only getting tighter. It is clear that we must continue to look carefully at how funding is best used and where we can get the maximum bang for our buck. I'm determined that NICE continues to operate in a completely evidence-based way and that we are transparent, open and trusted.}

\section{Q What kind of relationship do you want to have with industry?}

[Former NICE Chairman] Michael Rawlins and I spent a week together in the USA in March, and I was interested that pretty much every pharmaceutical and life sciences company that we met was very respectful of NICE. I don't think they were just being polite to us either. I think they genuinely respected the quality of the way that we work. I suspect the life sciences industries may not always like what we do - and I know that has been the case in the past - but I hope that they will at least respect us for doing a high-quality, difficult job with fairness and openness.

\section{Q How will the value-based pricing system, due to come into play by} January 2014, affect NICE appraisals? We are still waiting to find out. [The UK's Department of Health has not yet released details of the new pricing scheme.] We're very pleased, though, that NICE will be responsible for the full-value assessment under the future system. Many people tend to look at NICE as the organization that says "no", but in fact the vast majority [80-90\%] of the decisions we make are positive. My hunch would be that the new value-based pricing arrangements will ensure that more medicines will have a price that is a fair reflection of their value. I'm hoping it will be beneficial for everybody.

I sincerely hope the change will be evolutionary rather than revolutionary. I think it is going to be challenging to adopt the new system by January 2014, but we will do all we can to start appraisals by then.

Q In April, NICE took over responsibility for assessing the cost-benefit of drugs for rare diseases. Will such assessments require new methodologies?

We're looking at the moment at how to assess these. We've been discussing it with the board recently and will be putting our likely way forward for consultation out in due course.

A key question for me, though, comes down to how you merge thresholds. If you have one system to assess very rare conditions and another threshold for other conditions, is that the most appropriate way of doing things? Should there be a transition when you move between the different methodologies? The answer is, we don't know yet. The definition of very rare conditions is constantly changing as well. More work in genetics allows us to define some conditions as subsets of another: is that a very rare condition now? It is a complex area.

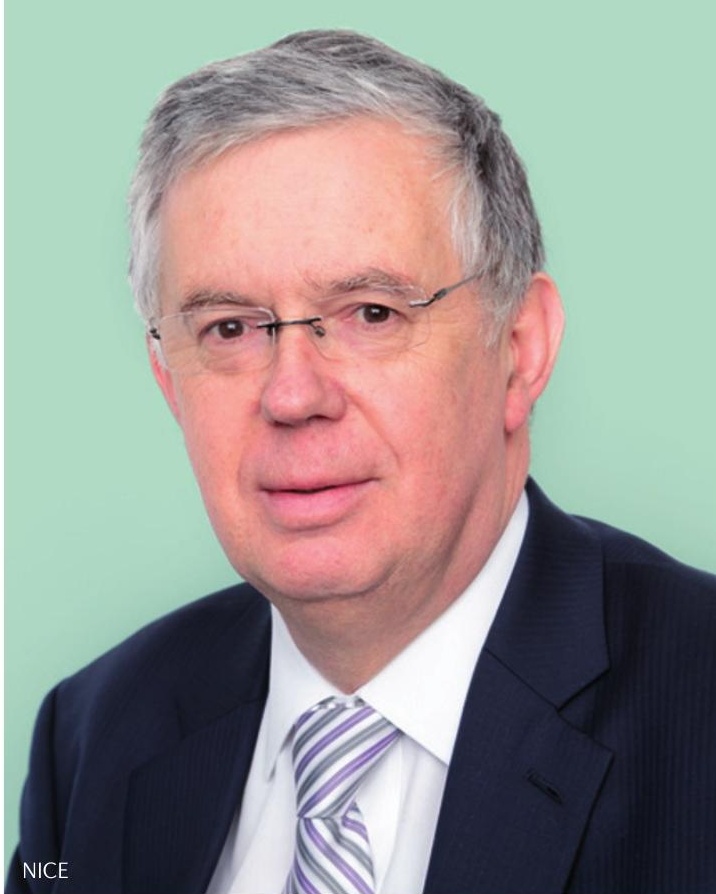

Q Are you going to develop stronger ties with other health technology assessment (HTA) organizations?

I'm sure we have to build better bridges. I absolutely loathe unnecessary duplication. There are obviously significant differences between the way different health-care systems work and the things that they prioritize. I hope there is much more that we could do together, but I'm far too new at this to tell you what that is.

\section{Q What other priorities do you have?} NICE has got a massively extended role, and our work now extends across health care, social care, quality standards, quality guidelines, and so on. I suspect one of the reasons why I was appointed is because of my experience as a generalist.

Fundamentally, my main concern is around multiple morbidity and comorbidity. We rarely treat patients with single conditions but rather treat patients with multiple long-term conditions, at which point the treatment guidances we provide become much more complex. For a patient with heart disease, chronic kidney disease, arthritis, depression and diabetes, doctors can't just add together all the guidances or the patient would have to take 50 pills a day and get blood tests twice a week. Defining quality for these patients is a major challenge that I am very keen to take on. The other major challenge we have is on setting social care standards, trying to bridge the gap between health and social care.

It's an extraordinarily broad remit now, from the care of patients with dementia in care homes right the way through to the complexity of HTA. 